\title{
Optical von Neumann measurement
}

\author{
G. M. D'Ariano and M. F. Sacchi \\ Dipartimento di Fisica "A. Volta", via Bassi 6, I-27100 Pavia, ITALY \\ DARIANO@PV. INFN. IT \\ MSACCHI@PV. INFN. IT
}

1993 PACS number(s): 42.50.-p, 05.30.-d, 02.90.+p

\begin{abstract}
We present an optical scheme that realizes the standard von Neumann measurement model, providing an indirect measurement of a quadrature of the field with controllable Gaussian state-reduction. The scheme is made of simple optical elements, as laser sources, beam splitters, and phase sensitive amplifiers, along with a feedback mechanism that uses a Pockels cell. We show that the von Neumann measurement is achieved without the need of working in a ultra-short pulsed regime.
\end{abstract}

In the last chapter of his book[1] von Neumann formulated a measurement scheme for the position $\hat{q}$ of a particle based on a coupling with another particle. The interaction Hamiltonian between the two particles - object and probe - is of the form $\hat{H}_{I}=\hat{q} \hat{P}$, product of the object's position $\hat{q}$ with the probe's momentum $\hat{P}$. It is switched on with a very strong coupling and for a very short time, and immediately afterwards a measurement of the probe-particle position $\hat{Q}$ is performed. By shifting the probe's position $\hat{Q}$ by anamount proportional to the object's position $\hat{q}$, the coupling correlates the object's position with the probe's "pointer observable" $\hat{Q}$, through which the object's position is obtained, thus leaving the particle available for a forthcoming measure.

Originally, von Neumann introduced his model in order to discuss the repeatability hypothesis suggested by the Compton-Simons experiment. After, it remained as a reference point for theoretical models of repeatable measurements, an ideal "gedanken microscope" with controllable disturbance on the system (see, for example, Ref. [2], where the von Neumann model is considered in relation to the problem of position measurements below the standard quantum limit).

Is it possible to achieve this model experimentally? As a particle Hamiltonian, the $\hat{q} \hat{P}$ interaction is rather artificial. However, we will show that in the domain

Preprint submitted to Elsevier Preprint 30 October 2018 
of quantum optics, one can achieve the von Neumann measurement (i. e. with the same probability distribution and the same "state-reduction") without the need of either realizing the precise form of the von Neumann Hamiltonian, or of experimentally achieving the impulsive limit: and from this prototype scheme for the von Neumann measurement we will have learned an interesting lesson useful for future quantum measurement engineering. The scheme we present is made of simple optical elements, as laser sources, beam splitters, and phase sensitive amplifiers, along with a feedback mechanism that uses a Pockels cell. We will employ a "pre-amplification" of the signal state and a "pre-squeezing" of the probe state: this is the basic idea to improve the quality of a quantum measurement [3], that has already been implemented in the realm of backaction evading measurements [4-7].

For a single mode of the radiation field, the optical observables that correspond to particle position $\hat{q}$ and momentum $\hat{p}$ are represented by any two conjugated quadratures $\hat{x}_{\phi}$, and $\hat{x}_{\phi+\pi / 2}$, with commutator $\left[\hat{x}_{\phi}, \hat{x}_{\phi+\pi / 2}\right]=i / 2$, the generic quadrature being defined as follows

$$
\hat{x}_{\phi}=\frac{1}{2}\left(a^{\dagger} e^{i \phi}+a e^{-i \phi}\right)
$$

The quadrature $\hat{x}_{\phi}$ can be ideally measured by means of a homodyne detector, in the limit of strong coherent local oscillator (LO), $\phi$ being the phase of the signal mode relative to the LO [8]. In Eq. (1), $a$ and $a^{\dagger}$ are the bosonic annihilation and creation operators of the field mode of interest, with commutation $\left[a, a^{\dagger}\right]=1$.

Now we need to settle the general theoretical framework for describing repeatable measurements. In order to have a measurement that does not completely destroy the state that the system had before the measurement, the scheme must involve a probe that interacts with the system and later is "measured" to yield information on the original state of the system [2]. This indirect measurement scheme is completely specified once the following ingredients are given: i) the unitary operator $\hat{U}$ that describes the system-probe interaction; ii) the state $|\varphi\rangle$ of the probe before the interaction; iii) the observable $\hat{X}$ which is measured on the probe. If, at the end of the system-probe interaction, one now considers another measurement on the system-say the ideal measurement of an observable $\hat{Y}$ (both $\hat{X}$ and $\hat{Y}$ have continuous spectrum, with eigenvectors $|x\rangle$ and $|y\rangle$, respectively), then the conditional probability density $p(y \mid x)$ - of getting a result $y$ from the second measurement given the result of the first one being $x$-can again be written in terms of the Born's rule $p(y \mid x) d y=\left\langle y\left|\hat{\varrho}_{x}\right| y\right\rangle$ upon defining a "reduced state" $\hat{\varrho}_{x}$ as follows 


$$
\hat{\varrho}_{x}=\frac{\hat{\Omega}(x) \hat{\varrho} \hat{\Omega}^{\dagger}(x)}{\operatorname{Tr}\left[\hat{\varrho} \hat{\Omega}^{\dagger}(x) \hat{\Omega}(x)\right]},
$$

where the system operator $\hat{\Omega}(x)$ is given by

$$
\hat{\Omega}(x)=\langle x|\hat{U}| \varphi\rangle,
$$

and

$$
d \hat{\mu}(x)=\hat{\Omega}^{\dagger}(x) \hat{\Omega}(x) d x
$$

is the "probability operator-valued measure" (POM) of the apparatus [2], which provides the Born's rule for the measurement as follows

$$
p(x) d x=\operatorname{Tr}[\hat{\varrho} d \hat{\mu}(x)] .
$$

Eqs. (2-5) are the most general form of the state-reduction and of the Born's rule for a "pure" or "quasi-complete" measurement, namely a measurement that leaves pure states as pure (due to the pure state preparation of the probe). Apart from an irrelevant phase factor, the non-unitary reduction operator $\hat{\Omega}(x)$ uniquely characterizes the quantum measurement, and two measurements that have the same operator $\hat{\Omega}(x)$ will be considered as identical, both having the same probability density (5) and the same state-reduction (2). On the other hand, the fact that many measurements can share the same POM $d \hat{\mu}(x)$ while having different state reduction - is immediately apparent from the fact that a unitary transformation of the reduction operator $\hat{\Omega}(x) \rightarrow \hat{\Omega}^{\prime}(x)=$ $\hat{V}(x) \hat{\Omega}(x)$ changes only the state-reduction, but leaves the POM (4) invariant. A unitary transformation $\hat{V}(x)$ that depends on the result $x$ of a measurement is the quantum mechanical description of a feedback mechanism, which in turn represents the easiest way of engineering a prescribed (admissible) state reduction.

In the above framework, the standard von Neumann measurement model is given by the Gaussian reduction operator

$$
\hat{\Omega}(q)=\left(\frac{1}{2 \pi \Delta^{2}}\right)^{1 / 4} \exp \left[-\frac{(q-\hat{q})^{2}}{4 \Delta^{2}}\right],
$$

which results from an impulsive interaction Hamiltonian $\hat{q} \hat{P}$ with the probeparticle prepared in a Gaussian wave packet. From Eqs. (4) and (5) it follows that the experimental probability density $p(q)=\operatorname{Tr}\left[\varrho \hat{\Omega}^{\dagger}(q) \hat{\Omega}(q)\right]$ is just a Gaussian convolution of the ideal probability distribution $\langle q|\hat{\varrho}| q\rangle$, with additional r.m.s. noise given by $\Delta$ (for interaction time $\tau=\kappa^{-1}$ with $\kappa$ the interaction 
strength, $\Delta^{2}$ is simply given by the variance of the probe-particle Gaussian wave-packet).

Now we present the quantum-optical scheme that performs the standard von Neumann measurement of the quadrature $\hat{x}_{\phi}$ of the radiation field in Eq. (1). In the following we will consider a fixed phase $\phi$, using the short notation $\hat{x}=\hat{x}_{\phi}, \hat{y}=\hat{x}_{\phi+\pi / 2}$. In a fully optical measurement scheme the simplest choice for a measuring probe is just another mode of the field. We consistently use capital letters for the probe operators: thus, $\hat{A}$ and $\hat{A}^{\dagger}$ will denote the annihilation and creation operators of the probe mode, whereas $\hat{X}$ and $\hat{Y}$ will be used to represent any couple of conjugated quadratures of the probe for fixed phase $\phi^{\prime}$. With this notation, the optical equivalent of the standard von Neumann Hamiltonian (for indirectly measuring the quadrature $\hat{x}$ by probing $\hat{X})$ is given by

$$
\hat{H}=\hat{x} \hat{Y}
$$

Notice that the choice of the phases $\phi$ and $\phi^{\prime}$ is totally free, and is ultimately related to the definition itself of the annihilation and creation operators of the two modes. From the definition (1) of $\hat{x}_{\phi}$ we can immediately see that, independently on the frequency of the two field modes, the Hamiltonian (7) cannot be realized in the rotating wave approximation, due to the counterrotating terms $\hat{A} \hat{a}$ and $\hat{A}^{\dagger} \hat{a}^{\dagger}$. On the other hand, an impulsive realization of this Hamiltonian, as in the original formulation of von Neumann, again is not feasible in the optical domain, because it would require switching the interaction faster than the optical frequency. However, as we will show in the following, we don't need to realize the Hamiltonian (7) in order to achieve the von Neumann measurement.

Instead of the Hamiltonian (7) we consider the interaction of the two field modes at a beam splitter. This is described by the unitary evolution operator [8]

$$
\hat{U}=\exp \left[\operatorname{atan} \sqrt{\frac{1-\eta}{\eta}}\left(a b^{\dagger}-a^{\dagger} b\right)\right]
$$

The unitary evolution operator (8) has no counter-rotating terms: in the following we will take both modes at the same frequency, so that the operator (8) will retain its time-independent form also in the interaction picture (the simple form of the operator (8) holds for an appropriate choice of the modal phases, which can be achieved by just changing optical path lengths). Expressed as a function of the field quadratures, the unitary operator $\hat{U}$ reads 


$$
\hat{U}=\exp \left[2 i \operatorname{atan} \sqrt{\frac{1-\eta}{\eta}}(\hat{y} \hat{X}-\hat{x} \hat{Y})\right] .
$$

The operator in Eq. (9) can be conveniently factorized into the product of elemental unitary evolutions by exploiting the realization of the $s u(2)$ algebra $\hat{J}_{+} \equiv 2 i \hat{y} \hat{X}, \hat{J}_{-} \equiv 2 i \hat{x} \hat{Y}, \hat{J}_{z} \equiv i(\hat{X} \hat{Y}-\hat{x} \hat{y})$, where one can easily verify the $s u(2)$ commutation relations $\left[\hat{J}_{+}, \hat{J}_{-}\right]=2 \hat{J}_{z},\left[\hat{J}_{z}, \hat{J}_{ \pm}\right]= \pm \hat{J}_{ \pm}$. Using the BCH formula for the $S U(2)$ group [9], the operator $\hat{U}$ can be written as follows

$$
\hat{U}=e^{2 i \sqrt{\frac{1-\eta}{\eta}} \hat{y} \hat{X}} \eta^{i(\hat{x} \hat{y}-\hat{X} \hat{Y})} e^{-2 i \sqrt{\frac{1-\eta}{\eta}} \hat{x} \hat{Y}} .
$$

The last factor on the right of Eq. (10) has the same form of the von Neumann unitary evolution for Hamiltonian (7). The physical meaning of other two factors will become clear after evaluating the reduction operator $\hat{\Omega}(x)$ corresponding to the unitary evolution in Eq. (10).

Let $|\varphi\rangle$ be the state preparation of the probe mode before the measurement, (the state of the field mode $A$ that enters one port of the beam splitter), and let us denote by $|x\rangle$ the eigenvectors of the quadrature $\hat{X}$ effectively measured at one output port of the beam splitter by means of a homodyne detector. Then, $\hat{\Omega}(x)$ can be evaluated through the following steps

$$
\begin{aligned}
& \hat{\Omega}(x)=\left\langle x\left|e^{2 i \sqrt{\frac{1-\eta}{\eta}} \hat{X} \hat{y}} \eta^{i(\hat{x} \hat{y}-\hat{X} \hat{Y})} e^{-2 i \sqrt{\frac{1-\eta}{\eta}} \hat{x} \hat{Y}}\right| \varphi\right\rangle \\
& =e^{2 i \sqrt{\frac{1-\eta}{\eta}} x \hat{y}} \eta^{i \hat{x} \hat{y}}\left\langle x\left|\eta^{-i \hat{X} \hat{Y}} e^{-2 i \sqrt{\frac{1-\eta}{\eta}} \hat{x} \hat{Y}}\right| \varphi\right\rangle \\
& =e^{2 i \sqrt{\frac{1-\eta}{\eta}} x \hat{y}} \eta^{i \hat{x} \hat{y}} e^{-\ln \eta^{1 / 2} x \partial_{x}} e^{-\sqrt{\frac{1-\eta}{\eta}} \hat{x} \partial_{x}} \varphi(x) \\
& =\hat{D}_{a}^{\dagger}\left(\sqrt{\frac{1-\eta}{\eta} x}\right) \hat{S}_{a}^{\dagger}\left(\ln \eta^{1 / 2}\right) \times \\
& \times \eta^{-1 / 4} \varphi\left[\eta^{-1 / 2}\left(x-(1-\eta)^{1 / 2} \hat{x}\right)\right],
\end{aligned}
$$

where $\hat{S}_{a}(r)$ and $\hat{D}_{a}(\alpha)$ denote the squeezing and displacement operators of the mode $a$, namely

$$
\hat{S}_{a}(r)=e^{-i r(\hat{x} \hat{y}+\hat{y} \hat{x})}, \quad \hat{D}_{a}(\alpha)=e^{\alpha a^{\dagger}-\bar{\alpha} a},
$$

and we used the quadrature differential representation

$$
\langle x|f(\hat{X}, \hat{Y})| \varphi\rangle=f\left(x,-\frac{i}{2} \partial_{x}\right) \varphi(x) ; \quad \varphi(x) \equiv\langle x \mid \varphi\rangle
$$


The squeezing and displacement unitary operators that appear in the last step of Eq. (11) represent an additional back action from the measurement, i. e. they just change the state-reduction by an additional unitary evolution, but they do not change the POM, which for the reduction operator (11) is given by

$$
\begin{aligned}
d \hat{\mu}_{\eta}(x) & =d x \hat{\Omega}^{\dagger}(x) \hat{\Omega}(x) \\
& =d x \eta^{-1 / 2}\left|\varphi\left[\eta^{-1 / 2}\left(x-(1-\eta)^{1 / 2} \hat{x}\right)\right]\right|^{2}
\end{aligned}
$$

For very high reflectivity at the beam splitter $\eta \rightarrow 0$ and with the probe prepared in the vacuum state $|\varphi\rangle \equiv|0\rangle$, Eq. (14) would approach the Gaussian von Neumann POM from Eqs. (4) and (6) with variance $\Delta=\sqrt{\eta} / 2$. However, the reduction operator (11) is still different from that in Eq. (6), and in order to make them equal we need to remove the squeezing and the displacement back-action terms. The displacement term is a unitary transformation that depends on the measurement outcome, and hence it can be compensated by an appropriate feedback device. On the other hand, the squeezing term can be balanced by an inverse squeezing transformation of the mode $a$ performed after the displacing feedback: this will be the last transformation on the mode $a$, and we will refer to it as back-squeezing. For vanishing $\eta$ one would need increasingly large back-squeezing, and it may be more convenient to compensate the vanishing $\eta$ by squeezing the probe state $|\varphi\rangle$. In fact, squeezing transforms the quadrature $\hat{x}$ as follows

$$
\hat{x} \rightarrow \hat{S}_{a}^{\dagger}(r) \hat{x} \hat{S}_{a}(r)=e^{r} \hat{x}
$$

Hence the factor $(1-\eta)^{1 / 2}$ in the POM (14) can be removed by pre-squeezing the initial state of the system with squeezing parameter $r=-\frac{1}{2} \ln (1-\eta)$. Such pre-squeezing modifies the reduction operator $\hat{\Omega}(x)$ in Eq. (11) into the following one

$$
\hat{\Omega}(x) \rightarrow \hat{\Omega}(x)=\eta^{-1 / 4} \varphi\left[\eta^{-1 / 2}(x-\hat{x})\right],
$$

where now we have changed the back-squeezing as follows

$$
\begin{aligned}
\hat{S}_{a}^{\dagger}\left(\ln \eta^{1 / 2}\right) & \longrightarrow \hat{S}_{a}^{\dagger}\left(\ln \eta^{1 / 2}\right) \hat{S}_{a}\left[\ln (1-\eta)^{-1 / 2}\right] \\
& =\hat{S}_{a}^{\dagger}\left[\frac{1}{2} \ln (\eta(1-\eta))\right]
\end{aligned}
$$

Then, in order to get a tunable variance for the reduction operator, one can change the state preparation $|\varphi\rangle$ of the probe. For the squeezed vacuum 


$$
|\varphi\rangle=\hat{S}_{A}\left(\ln \sigma^{1 / 2}\right)|0\rangle,
$$

the reduction operator (16) becomes

$$
\hat{\Omega}(x)=\left(\frac{2}{\pi \eta \sigma}\right)^{\frac{1}{4}} \exp \left[-\frac{(x-\hat{x})^{2}}{\eta \sigma}\right],
$$

and the operator $\hat{\Omega}(x)$ in Eq. (19) is of the same form of the von Neumann one in Eq. (6), with $\Delta=\sqrt{\eta \sigma} / 2$.

The experimental set-up to perform the optical von Neumann measurement is sketched in Fig. 1.

Fig. 1. Outline of the proposed experimental setup to realize a von Neumann measurement of a quadrature of the electromagnetic field. BS denote a beam splitter; $\operatorname{PC}(\theta)$ denotes a Pockels cell with transmissivity $\theta$.

The pre-squeezing and back-squeezing transformation described by the unitary operators

$$
\hat{S}_{a}^{1}\left[-\frac{1}{2} \ln (1-\eta)\right], \quad \hat{S}_{a}^{2}\left[\frac{1}{2} \ln (\eta(1-\eta))\right],
$$

are the two extremal steps of the sequence of optical operations on the system mode. They can be accomplished by two phase-sensitive amplifiers (PSA) [10] with gains $G_{1}=(1-\eta)^{-1}$ and $G_{2}=\eta(1-\eta)$, respectively. [The PSA ideally amplifies the quadratures of the field with a phase-dependent gain, namely $\hat{x}_{\phi}^{\prime}=G^{-1 / 2} \hat{x}_{\phi}, \hat{x}_{\phi+\pi / 2}^{\prime}=G^{1 / 2} \hat{x}_{\phi+\pi / 2}$. It can be attained through degenerate three- or four-wave mixing.] In the same way the probe state (18) can be achieved using a third PSA that amplifies an input vacuum field with gain $G_{3}=\sigma$. After the first squeezing, the state of the $a$ mode is entangled with the squeezed vacuum state (18) of the $A$ mode through the beam splitter 
with transmissivity $\sqrt{\eta}$, and at the reflected output beam the quadrature $\hat{X}$ is homodyne detected. The displacement $\hat{D}\left(\sqrt{\frac{1-\eta}{\eta}} x\right)$ is achieved by combining the transmitted beam with a strong coherent LO $|\beta\rangle(\beta \rightarrow \infty)$ in a beam

splitter with a transmissivity $\theta \rightarrow 1$, such that $|\beta| \sqrt{1-\theta}=\sqrt{\frac{1-\eta}{\eta}} x$ (the LO is at the frequency of the signal mode $a$ ). The parametric dependence on the homodyne outcome $x$ is carried out by driving the LO with the homodyne photocurrent, for example by stimulating the laser that provides the LO by the photodetection current itself. However, this method is expected to fail for small "photocurrents" $x$, because it would bring the LO laser below threshold, thus loosing the phase of $\beta$. A better way to achieve this feedback is to provide a current-dependent transmissivity $\theta(x)$ for the beam-splitter, making use, for example, of a Pockels cell, and working in the linearity regime $\theta \propto x$ of the cell [a similar feedback mechanism has been experimentally implemented in Ref. [11]]. Of course, good phase coherence between the PSA pumps and the LO may be technically difficult to achieve. Finally, also notice that the quadrature phase $\phi$ can be changed in many different ways by tuning any one of the relative phase-shifts between the pumps and the LO.

In conclusion, we have presented a quantum-optical scheme that realizes the standard von Neumann model, a model for repeatable quantum measurements with controlled state-reduction. Our scheme uses simple optical elements, like beam-splitters and squeezers. We have seen that, contrarily to the customary modeling of repeatable measurements, there is no need of working in a ultra-short pulsed regime. We have also seen how the precise form of the state reduction can be engineered by means of a feedback mechanism that uses a Pockels cell: we think that this method can be of use in more general situations, for controlling the back-action of a quantum measurement. Finally we hope that our scheme will be implemented experimentally, and will be of help for a deeper understanding of the physical mechanisms underlying quantum measurements.

\section{References}

[1] J. von Neumann, Mathematical Foundations of Quantum Mechanics (Princeton Univ. Press, Princeton, NJ, 1955), pp. 442-445.

[2] M. Ozawa, in Squeezed States and Nonclassical Light, ed. by P. Tombesi and E. R. Pike, pag. 263, Plenum, (New York, 1989).

[3] J. H. Shapiro, Opt. Lett. 5, 351 (1980).

[4] A. La Porta, R. E. Slusher, and B. Yurke, Phys. Rew. Lett. 62, 28 (1989).

[5] Z. Y. Ou, S. F. Pereira, and H. J. Kimble, Phys. Rew. Lett. 70, 3239 (1993). 
[6] S. F. Pereira, Z. Y. Ou, and H. J. Kimble, Phys. Rew. Lett. 72, 214 (1994).

[7] K. Bencheikh, J. A. Levenson, Ph. Grangier, and O. Lopez, Phys. Rew. Lett. 75, 3422 (1995).

[8] For a tutorial review on concepts of quantum measurements and applications to quantum optics, see: G. M. D'Ariano, Quantum Estimation Theory and Optical Detection, in Concepts and Advances in Quantum Optics and Spectroscopy of Solids, ed. by T. Hakioğlu and A. S. Shumovsky. (Kluwer Academic Publishers, Amsterdam 1996, in press)

[9] Notice that the fact that our realization of the $s u(2)$ algebra does not preserve hermitian conjugation is irrelevant for the group multiplication, as in the $\mathrm{BCH}$ formula we are actually using the complexification of the group.

[10] H. P. Yuen, Phys. Lett. A 113, 405 (1986); Opt. Lett. 12, 789 (1987).

[11] F. De Martini, L. De Dominicis, E. Del Re, and F. Truc, in Quantum interferometry, ed. by F. De Martini, G. Denardo, A. Zeilinger (World Scientific, London 1995) pag. 210. 


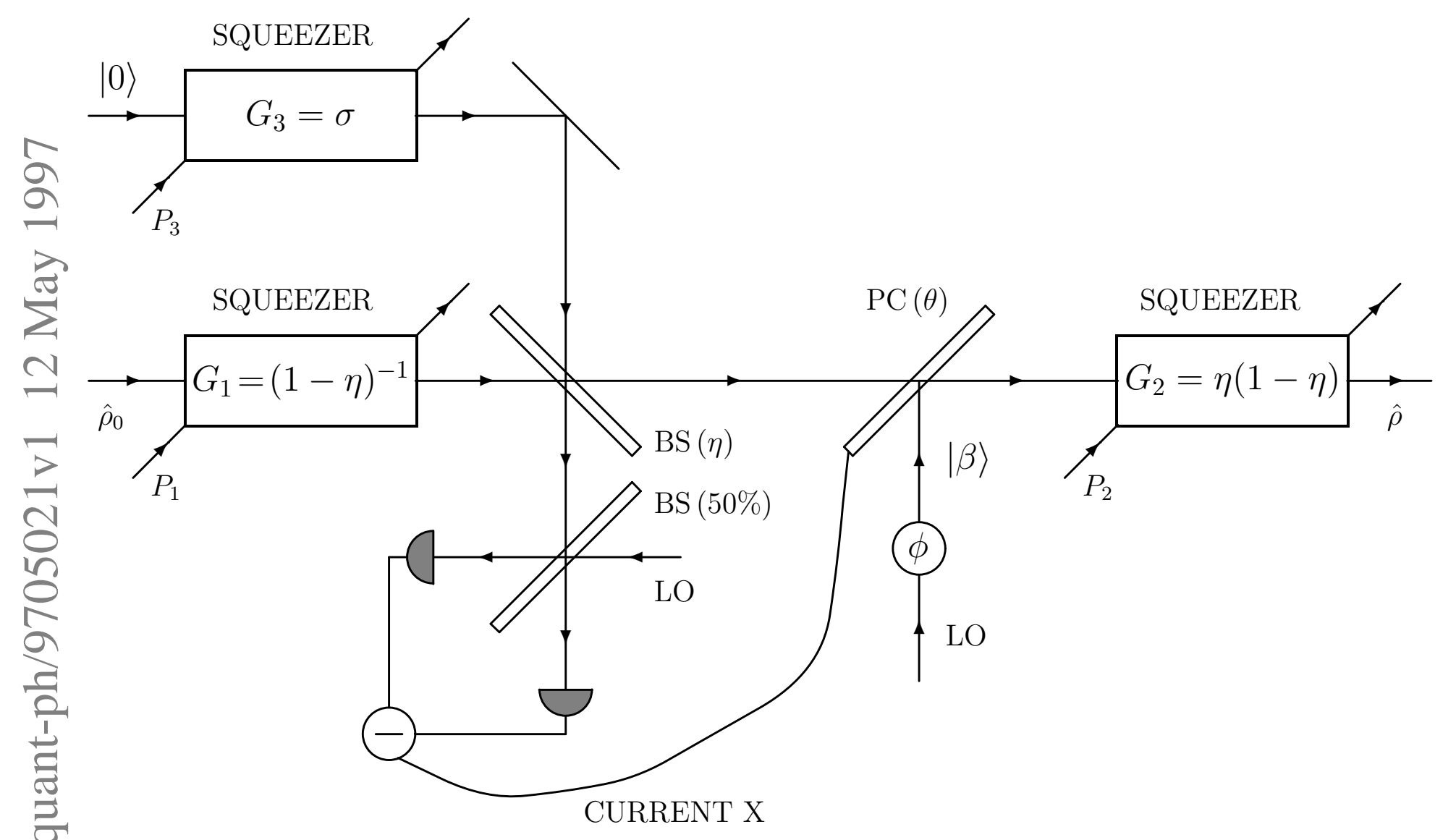

